\title{
Subjectivité et projection : le cas des particules discursives
}

\author{
Mathilde Dargnat* \\ Université de Lorraine et ATILF-CNRS, 44 avenue de la Libération, 54000 Nancy, France \\ Institut des Sciences Cognitives Marc Jannerod-CNRS, 67 boulevard Pinel, 69500 Bron, France
}

\begin{abstract}
Résumé. Cet article traite des particules discursives, qui sont des lexèmes ou expressions complexes figées connectés à la situation d'énonciation et au locuteur (bon, en fait, tiens, tu m'étonnes, quoi, zut, etc.). Elles sont abordées ici sous un angle relativement nouveau dans le domaine de l'étude des marqueurs de discours, celui de leur comportement vis-à-vis de la projection. La projection, problème connu en sémantique, désigne le fait que certains opérateurs (la négation, l'interrogation, les modaux de possibilité) n'affectent qu'une partie du contenu de l'énoncé dans lequel ils se trouvent. Un élément se projette s'il n'est pas affecté par ces opérateurs, l'exemple couramment donné étant celui des présuppositions. Les particules se comportent comme des éléments systématiquement projectifs. L'hypothèse est que cela est dû à leur nature présuppositionnelle et à la subjectivité inhérente à leur profil énonciatif.
\end{abstract}

\begin{abstract}
Subjectivity and projection : the case of particles. This paper deals with French discourse particles, that is, words or frozen complex expressions anchored to the utterance situation and to the speaker (bon, en fait, tiens, tu m'étonnes, quoi, zut, etc.). The perspective I adopt is relatively rare in the field of discourse markers studies. I focus on their behavior with respect to projection. Projection is a well-known problem in semantics. It refers to the fact that some operators (negation, question, possibility modals) do not affect the whole content of their host-uterrance. Some part of the semantic content is said to project whenever it is not affected by such operators. Presuppositions are a prototypical case. Particles behave like inherently projective items. I claim that this is due to their presuppositionnal status and to their necessary association with the speaker, which derives from their specific enunciative profile.
\end{abstract}

\section{Introduction}

Cet article pose le problème de la projection en général et plus spécifiquement de la projection des particules discursives. L'hypothèse est que leur propriété de projection est lié à leur profil énonciatif, et plus spécifiquement à leur ancrage subjectif.

La section 2 fait le point sur la notion de projection et sur les phénomènes qui ont été invoqués pour l'analyser, à savoir le focus et la question en discussion (QUD $\left.{ }^{1}\right)$. J'y discute

mathilde.dargnat@univ-lorraine.fr 
la conception classique de la projection (§ 2.1) appliquée aux présuppositions. Ensuite, je présente trois révisions majeures de cette conception $(\S 2.2)$ touchant : aux déclencheurs de présupposition ( $\S 2.2 .1$ ), aux opérateurs généralement utilisés pour tester la projection (§ 2.2.2), et aux arguments avancés pour expliquer les observations (§ 2.2.3).

La section 3 présente la classe des particules discursives $(\$ 3.1)$ et les examine du point de vue de la projection (\$3.2). Deux problèmes définitoires principaux sont développés : le fait qu'elles appartiennent à une classe fonctionnelle ouverte (§ 3.1.1) et la diversité des valeurs sémantiques $(\$ 3.1 .2)$. L'étude du comportement des particules vis-à-vis de la projection amène à avancer leur composante présuppositionnelle et à en pointer les conséquences (§3.2.1) ainsi qu'à souligner la différence avec une autre sous-classe de marqueurs de discours, les connecteurs $(\S 3.2 .2)$.

\section{La question de la projection}

\subsection{Conception « classique »}

Le problème de la projection ${ }^{2}$ n'est pas un problème nouveau en linguistique (cf. Frege 1892, Karttunen 2016, Langendoen \& Savin 1971, Morgan 1969). Il est au centre de nombreux travaux en sémantique et soulève, dans certains, la question des relations avec la pragmatique. Dans le détail, le phénomène de projection est assez complexe. Dans un premier temps, il peut être défini comme le fait que certains opérateurs exprimant la négation (ne...pas, douter que, etc.), l'interrogation (est-ce que..., etc.) ou la modalisation (peut-être que, il se peut que, si... etc.) n'affectent qu'une partie de l'énoncé dans lequel ils agissent. On dit que les éléments non affectés se projettent. Ils sont hors de la portée desdits opérateurs. On parle de projection en référence à une présentation arborescente, dans laquelle le contenu hors de la portée de l'opérateur se projette hors de l'arbre représentant l'énoncé. C'est typiquement le cas des présuppositions, comme dans (1), des expressifs ${ }^{3}$ (Potts 2005, 2007) comme dans (2), et des éléments attachés à l'état émotionnel ou épistémique du locuteur, comme dans $(3,4)$. Les déclencheurs de projection sont en italiques dans les exemples. Les opérateurs sont la négation de phrase dans ( $1 \mathrm{~b}, 3 \mathrm{~b}, 4 \mathrm{~b})$ et la construction interrogative dans $(2 b)$.

a. Paul a arrêté de fumer.

b. Paul n'a pas arrêté de fumer.

(2) a. Ton connard de voisin vient cet été.

b. Est-ce que ton connard de voisin vient cet été ?

(3) a. Il a hélas compris ce que je voulais dire.

b. Il n'a hélas pas compris ce que je voulais dire.

a. Il viendra demain, tu parles!

b. Il ne viendra pas demain, tu parles!

Dans (1a), le fait que Paul fumait avant est présupposé, et la présupposition se projette en (1b) (n'est pas atteinte par la négation). En (2a), le fait que Paul soit présenté comme un connard n'est pas remis en question en (2b). Le contenu de l'adverbe hélas en (3a) se projette en (3b), puisqu'il n'est pas atteint par la négation. Il est indépendant du contenu principal de l'énoncé, il indique le statut émotionnel (regret) du locuteur relativement au contenu de l'énoncé. L'expression figée tu parles en (4a) équivaut à une interjection et relève à ce titre de la classe des particules discursives (voir § 3.1). Elle signifie ici l'évidence et le non-étonnement du locuteur relativement au contenu de l'énoncé qui précède, avec semble-t-il une nuance de désapprobation. Tout comme hélas, tu parles peut être en position initiale ou finale.

Cette description classique fait de la projection un phénomène essentiellement lexicogrammatical. Historiquement, la plupart des nombreux travaux consacrés à la présupposi- 
tion au cours des trente dernières années du $20^{\mathrm{e}}$ siècle acceptent cette vision. La projection est définie comme ce que l'on observe lorsque l'on combine avec un opérateur (négation, question, verbe modal de possibilité) une phrase comportant un lexème ou une construction particulière. Le lexème ou la construction concernés sont dénommés déclencheurs (de présupposition) (triggers). La version la plus répandue de la projection peut se résumer schématiquement comme en (5), que je couple avec un exemple pour la clarté. Pour une présentation typique, voir l'ouvrage de Chierchia \& McConnell-Ginet (1990).

(5) Phrase P1 comportant un déclencheur, P1 présuppose la proposition $\mathrm{p}$

$\mathrm{P} 1$ = Marie sait que Paul fume

déclencheur = savoir que

$\mathrm{p}=$ Paul fume

Phrase P2 obtenue en appliquant un opérateur à P1, P2 présuppose la proposition $\mathrm{p}$

$\mathrm{P} 2$ = Marie ne sait pas que Paul fume

opérateur $=n e \ldots$ pas

$\mathrm{p}=$ Paul fume

Cette formule « Déclencheur + Opérateur $\rightarrow$ Projection » repose sur trois pivots dont des analyses plus détaillées ont montré, et ce même pendant la période " classique " (globalement 1970-2000), qu'ils n'étaient pas aussi robustes que l'on pouvait le penser. J'indique ci-dessous les aspects les plus importants de cette révision de la formule théorique ci-dessus.

\subsection{Principales révisions de la conception classique}

Trois aspects sont discutés, à savoir la catégorie du déclencheur (§ 2.2.1), l'effet de l'opérateur $(\S 2.2 .2)$ et l'explication des observations problématiques pour l'approche classique, avec l'introduction du problème de la détermination de la ligne principale du discours $(\S 2.2 .3)$.

\subsubsection{Le déclencheur}

La catégorie du déclencheur est variable et ne se résume pas aux déclencheurs de présupposition. Potts (2005, §2.4-2.5) défend l'idée qu'il existe également des déclencheurs d'implicatures conventionnelles, c'est-à-dire de propositions qui, comme les présuppositions, sont associées au code de la langue concernée (conventionnelles), mais qui se distinguent de ces dernières sur cinq points. Je n'en retiens que deux, les plus pertinents ici.

(a) Le statut vériconditionnel d'une implicature conventionnelle n'a pas d'effet notable sur celui de la phrase qui la déclenche (independance of truth value). Par exemple, en (2a), que le voisin mérite ou non le jugement insultant du locuteur n'a pas d'effet sur la vérité ou la fausseté du reste de la phrase (ton voisin vient cet été). Ce n'est pas (toujours) le cas pour les présuppositions. Par exemple, en (1a), si Paul n'a jamais fumé, la phrase peut être jugée fausse ou, en tout cas, inadéquate.

(b) Les implicatures conventionnelles sont liées au locuteur (speaker oriented) et, de ce fait, ne peuvent être attribuées à un autre agent de discours. Par exemple, dans une variante (2c) de (2) :

\section{(2c) Paul pense que ton connard de voisin vient cet été}

l'insulte est attribuée au locuteur et pas à Paul. Il y a ici une analogie avec la projection, en ce sens qu'un opérateur de type $x$ pense que ne modifie pas l'attribution d'une proposition. Cette question est rediscutée plus loin $(\S 3.2 .1)$. 


\subsubsection{L'opérateur}

Le deuxième aspect de la révision de la théorie classique concerne l'effet de l'opérateur. Dès les travaux de Kiparsky \& Kiparsky (1971) et de Karttunen (1971) sur les verbes dits factifs $^{4}$, il a été noté que, dans certains cas, un même opérateur peut entraîner ou ne pas entraîner de projection selon le déclencheur. C'est ce que montrent les exemples ci-dessous avec l'opérateur conditionnel si :

(6) Si Paul arrête de fumer, ...

(7) Si Paul découvre qu'il se trompe, il le reconnaîtra.

Dans (6), la proposition conditionnelle présuppose que Paul fume, mais, dans (7), la proposition conditionnelle ne présuppose pas nécessairement que Paul s'est trompé. D’une manière générale, il semble que la projection, même si elle est très fréquente, ne soit pas une propriété systématique et qu'elle doive s'analyser plutôt en termes de degrés (Tonhauser et al. 2018).

\subsubsection{L'explication}

L'explication de cet ensemble d'observations correspondant à la projection est la question la plus délicate, car il est difficile d'évaluer les différentes approches sur une base empirique solide. Je laisse de côté pour le moment l'approche dite anaphorique (van der Sandt 1992, Geurts 1999) sur laquelle je reviendrai dans la section 3.2.1. Je me concentre ici sur trois approches, celle de Beaver et al. (2017), de Ducrot (1972) et de Stalnaker (1974). Malgré leurs différences, ces approches partagent une intuition commune : les présuppositions (Ducrot, Stalnaker) et, plus largement, les propositions qui se projettent (présuppositions ou autres), sont présentées comme en dehors de la ligne principale du discours. Cette ligne peut être appréhendée comme ce qui, dans la contribution d'un locuteur, sert à répondre à une question implicite ou explicite. Le terme de question ne renvoie pas ici nécessairement à une phrase interrogative, mais, de manière plus abstraite, à un thème autour duquel un débat peut s'organiser. Une partie de la littérature anglosaxonne utilise dans ce cas l'expression de Question Under Discussion (QUD) (voir entre autres Ginzburg 2012, Roberts 2012). La projection s'observe lorsqu'un opérateur cible la ligne principale et, du même coup, laisse de côté ce qui n'appartient pas à cette ligne, en d'autres termes ce qui ne contribue pas à éclaircir la QUD (présuppositions et implicatures, en particulier). La plupart des approches admettent que la portée des opérateurs, c'est-àdire le fait qu'ils ciblent ou non la ligne principale, est déterminée de façon conventionnelle. La portée fait partie de la manière dont nous apprenons l'usage dominant de tels opérateurs. Cela rejoint la dimension lexico-grammaticale de la description classique des présuppositions.

L'unité relative des trois approches mentionnées disparaît lorsque l'on aborde la question de la détermination de la ligne principale. Qu'est-ce qui fait, dans un discours, que tel contenu fait partie de la ligne principale ou pas ? Est-ce le code linguistique qui guide notre interprétation en marquant un contenu comme principal ou secondaire ? Les approches qui n'utilisent pas la QUD (Ducrot, Stalnaker) tendent à privilégier cette réponse. Celles qui reposent sur la QUD favorisent un autre scénario, beaucoup plus pragmatique : c'est en fonction de l'interprétation la plus plausible en contexte que sont distribués les rôles. Pour une QUD donnée, les interprétations prioritaires sont celles qui permettent de rendre un mouvement conversationnel pertinent par rapport à cette QUD, c'est-à-dire de le lire comme une contribution plausible au thème du débat, que ce thème soit explicite ou implicite. Cela implique que le marquage linguistique institue au mieux une préférence par défaut. Ainsi, dans une phrase comme (8),

(8) Marie a découvert que Paul avait un entretien d'embauche. 
la structure factive présente par défaut la proposition Paul avait un entretien d'embauche comme hors de la ligne principale, alors que la connaissance par Marie du fait que Paul avait un entretien d'embauche est un élément de la ligne principale. En revanche, si la phrase constitue une réponse à la question « Qu'est-ce que Paul faisait hier ? », c'est le fait que Paul avait un entretien d'embauche qui peut sembler constituer la réponse à la QUD, l'état mental de Marie par rapport à ce fait n'ayant qu'un rôle secondaire (Simons 2007). Simons et al. (2011, 2017), ainsi que Beaver et al. (2017), proposent différentes présentations de cette approche pragmatique et contextuelle, qui est discutée de manière critique par Jayez (2010), Karttunen (2016), Peters (2016) et Dargnat \& Jayez (sous presse).

Dans la section suivante, je reviens sur la première question, celle du statut des déclencheurs. Je note d'abord une sorte de paradoxe. Alors qu'il existe une littérature abondante sur les marqueurs de discours, la question de leur statut quant à la projection est rarement abordée. Or il existe une sous-classe importante de ces marqueurs, désignés ici par le terme passe-partout de particules discursives, qui conjoignent deux propriétés : (a) de par leur indexicalité ils sont présuppositionnels ; (b) ils se projettent obligatoirement. Ces particules constituent en fait, comme on le verra, une catégorie nouvelle par rapport à l'approche de Potts et ne se laissent pas ramener à une analyse pragmatique de la projection.

\section{Spécificité des particules discursives}

Je liste d'abord quelques paramètres pour délimiter cette sous-classe de marqueurs de discours $(\S 3.1)$. Je me concentre sur la difficulté de circonscrire les propriétés de la classe particules discursives et d'établir une liste de ses éléments lexicaux (\$3.1.1), et sur les effets que ces particules produisent, c'est-à-dire leur sens en contexte (\$ 3.1.2). Ensuite, je reviens sur l'objet principal de la présente analyse, à savoir le comportement de ces particules vis-à-vis de la projection $(\S 3.2)$. Ceci permet de reprendre la question de l'approche anaphorique des présuppositions (\$ 3.2.1) et d'insister sur la différence de comportement entre deux sous-classes de marqueurs de discours proches, les connecteurs et les particules discursives $(\S 3.2 .2)$.

\subsection{Paramètres définitoires}

Les particules discursives désignent ici des lexèmes ou des expressions complexes figées connectés à la situation d'énonciation et au locuteur. Elles fonctionnent de manière déictique dans le discours et l'interaction. On les trouve généralement à l'oral, mais aussi dans les écrits dialogiques et/ou spontanés. Au cours des dernières décennies, elles ont fait l'objet de nombreuses études dans des langues diverses, plus ou moins ciblées sur des items, des propriétés ou des processus (Aijmer 2006, Beeching 2001, 2016, Denturck 2008, Dostie 2004, Fernandez-Vest 1994, Fischer 2006, Fraser 1999, Hansen 1998, Traugott 1982, Vincent 1993, Vincent \& Sankoff 1992, Bastien et al. 2016, Bartkova et al. 2017, etc.). Les terminologies, les cadres théoriques et les propriétés mises en avant ne sont pas toujours clairement superposables ni même comparables, mais on peut tout de même dégager quelques constantes - qui ne sont pas sans poser problème - qui serviront de paramètres définitoires (pour une synthèse et une discussion plus complètes, voir Dargnat sous presse). Le terme lui-même de particule, discursive ou énonciative, n'est pas systématique, puisque l'on trouve petite marque du discours, pragmatème, marqueur pragmatique, ponctuant de la langue, etc. Il est cependant important de considérer qu'il s'agit d'une sous-classe de la classe plus générale des marqueurs de discours, qui comporte aussi, entre autres, les connecteurs, dont le fonctionnement diffère des particules, notamment pour ce qui est de l'orientation sur le locuteur et l'effet local (voir $\S 3.2 .2$ ). 


\subsubsection{Classe ouverte et multi-catégorielle}

Cette classe se compose de lexèmes ou d'expressions complexes figées, et il est difficile de proposer une liste d'items et de leur emploi exclusif ou non comme particules. Ceci est pertinent pour la lexicographie en particulier (sur le français, voir entre autres Dostie 2004, Métrich et al. 2002) et pour les recherches en corpus d'une manière générale. Par exemple, que doit-on rechercher dans les données de corpus quand on veut étudier les particules discursives ? Dans l'abondante littérature les concernant, celles-ci ne sont généralement pas présentées comme une catégorie grammaticale au même titre que les noms, les adjectifs, etc. mais plutôt comme une classe fonctionnelle, c'est-à-dire identifiable à partir de son rôle en discours (pour une approche différente, voir Paillard 1998). Ainsi, dans l'ensemble des particules, on trouve des éléments par ailleurs classés comme adjectifs (bon, chic, etc.), adverbes (alors, bien, là, etc.), conjonctions (mais, etc.), noms (ciel, merde, pardon, etc.), verbes (allez, écoute, regarde, tiens, etc.), pronom (quoi). On trouve aussi des syntagmes nominaux (la vache, ma foi, ma parole, etc.), des syntagmes prépositionnels (à bientôt, à la fin, en fait, etc.), des syntagmes phrastiques (je veux dire, tu parles, vous voyez, etc.) et des combinaisons diverses comme bon ben (alors), eh bien, mais enfin, etc. Pour simplifier, je considère ici que les interjections sont un sous-ensemble des particules, spécialisé dans l'expression des émotions du locuteur (ah, ouf, zut, etc.). Il existe sur cette question plusieurs perspectives, dont le développement n'est pas nécessaire pour mon étude.

\subsubsection{Fonction des particules}

Les fonctions des particules sont apparemment très diverses et cumulables. Le plus souvent, elles se présentent comme manifestant :

- La réaction émotionnelle du locuteur à un événement externe ou interne (oh, tiens, etc.). On retrouve ici toutes les interjections et les particules expressives, comme en (9) et (10) :

Le locuteur laisse tomber un verre, qui se casse. - Zut !/Merde !/Mince! Youpi ! C'est Noël ! (youtube, 17/12/10)

En (9), zut, merde ou mince expriment toutes une émotion négative, par exemple la colère, le mécontentement, le regret, etc. en réaction à un événement. En (10), youpi exprime la joie du locuteur en réaction à une situation qui est explicitée dans la suite.

- Le positionnement épistémique du locuteur. Euh, disons, tiens, etc. peuvent par exemple marquer l'hésitation, l'approximation, la perception de la nouveauté ou du caractère évident d'une information. En (11), tiens permet au locuteur de présenter la cause introduite par parce que comme une évidence :
A : Pourquoi il l'aimait à ce point ?
B : Parce que c'était Chet Baker, tiens ! Un immense musicien ! (A.
Gavalda, La consolante, 2008)

On comprend aussi qu'ainsi le locuteur impose en même temps sa perception à l'interlocuteur, et que la fonction ci-dessous est également associée à tiens dans ce contexte.

- L'intention du locuteur d'orienter l'attention de l'interlocuteur ou d'influencer ses perspectives. Se retrouvent ici bon nombre de particules déverbales comme regarde/z, remarque/z, écoute/z, mais aussi des expressions figées comme point barre en (12).

«Une femme est l'égale de l'homme, point barre» a dit Julie. (V. Linhart, Le jour où mon père s'est tu, 2008)

L'expression point barre, en renforçant la valeur illocutoire de l'énoncé, permet au locuteur d'imposer son point de vue (il ne souhaite pas discuter ce qu'il déclare). De ce fait, il coupe court à la discussion. Point barre sert alors à la fois à guider l'interprétation de l'énoncé et aussi à gérer l'interaction ${ }^{5}$, comme le fait bon ci-dessous. 
- L'emploi de particules permet au locuteur de gérer l'interaction elle-même, à différents niveaux, pour exprimer une suggestion, une demande, effectuer une relance, interrompre le discours en cours, le sien ou celui de son interlocuteur. C'est typiquement ce que fait bon en (13) :

MP je l'ai dit je l'ai dit pour me moquer

PG vous êtes tellement vous êtes tellement mortifère pour la littérature que je vais pas

BP oui \# bon \# Michel Polac Michel Polac

PG vous laisser vous moquer c'est beau un adjectif

MP bon : bon

BP bon attendez bon (enregistrement d'Apostrophes avec Bernard Pivot, Patrick Grainville et Michel Polac, dans Brémond 2002, ex. 28, p. 434).

Face à la joute verbale engagée entre Michel Polac (MP) et Patrick Grainville (PG), le présentateur de l'émission, Bernard Pivot (BP), et Patrick Grainville lui-même tentent à plusieurs reprises d'interrompre les échanges au moyen de bon, qui a comme équivalent : arrêtons/arrêtez de parler.

Malgré cette diversité, les particules remplissent une fonction homogène qui peut se décliner en deux points.

(a) Les particules font nécessairement référence à leur propre situation d'énonciation et en sélectionnent certains éléments qui se manifestent dans cette situation. Parmi ceux-ci, les plus évidents sont les participants ou les événements non discursifs (un événement physique qui se produit dans le contexte), les événements interactionnels (typiquement le discours ou le comportement de l'interlocuteur, mais dans certains cas du locuteur luimême), les événements psychologiques internes (prise de conscience, étapes d'un raisonnement, variations de saillance et d'attention, etc.). Une particule peut renvoyer à plusieurs éléments simultanément. Par exemple, l'usage de pardon pour s'excuser d'avoir bousculé quelqu'un renvoie au comportement du locuteur (événement) et à l'interlocuteur présent (participant).

(b) Les particules ne se contentent pas de pointer sur ces paramètres, mais les présentent ou les "décrivent» sous un certain angle ou interagissent avec eux. Il est clair que l'interaction est ancrée dans la situation d'énonciation. C'est le cas de pardon mentionné cidessus et de toutes les particules qui ont une fonction d'adresse, même si elles ne s'y réduisent pas. En revanche, il y a beaucoup de cas moins transparents, dont les interjections. Il a été noté à plusieurs reprises qu'une interjection n'est pas la description d'un état psychologique. Dire Aïe ! et je me suis cogné n'est pas équivalent (Ducrot 1984, Kaplan 1999, Wharton 2003). Les interjections se présentent comme "arrachées par la situation » (Ducrot 1984). Ce qui est spécifique de l'interjection n'est pas tant son contenu descriptif que le fait qu'elle fait référence obligatoirement à un élément que l'on ne peut transporter hors de la situation d'énonciation. Le locuteur peut s'être cogné hier, mais il ne peut pas construire une combinaison telle que Aïe hier! Il faut donc préciser que, lorsqu'une particule a un contenu descriptif, ce contenu ne peut s'appliquer qu'à un objet ou une entité qui sont obligatoirement contemporains de la production de la particule. La «situation d'énonciation» doit donc être comprise comme la situation ancrée temporellement à l'énonciation de la particule. Les expressifs au sens de Potts se comportent différemment : ils ont un contenu descriptif, et peuvent pointer vers un élément contemporain de leur énonciation, mais ce n'est pas obligatoire, comme le montre la traduction de l'exemple suivant :

(14) J'ai vu presque tous les fichus (bloody) sketchs des Monty Python! (d'après Potts 2005, 2.21a, p. 8) 
En (14), l'événement que constitue le visionnage des sketchs n'est pas contemporain de l'énonciation de l'expressif fichu (bloody).

Il y a donc deux dimensions dans la constitution d'une particule : la dimension déictique, c'est-à-dire le verrouillage de la particule par la situation d'énonciation et la dimension sémantique, qui correspond aux conditions d'emploi de la particule (son sens général, qui peut se spécifier en contexte). La combinaison de ces deux dimensions constitue la spécificité de la classe. A titre de comparaison, les pronoms et déterminants déictiques ( $j e, t u$, mon, vos, etc.) sont liés à la situation d'énonciation, mais ne servent qu'à localiser les référents. Les particules possèdent en plus la dimension sémantique qui présente les référents d'une certaine manière, ou comme éléments d'un certain scénario.

En tant que liées à la situation d'énonciation, les particules relèvent des éléments qui ont une signification use-conditional dans la terminologie de Gutzmann (2013, 2015, 2019), c'est-à-dire qui sont des éléments dont les conditions d'emploi ne sont pas équivalentes aux conditions de vérité des phrases, même si elles peuvent y contribuer. Dans la classification qu'il propose, les particules n'ont aucun rôle vériconditionnel et s'appliquent à des propositions, vis-à-vis desquelles elles constituent des opérateurs. Vu ce statut d'opérateurs, Gutzmann estime que les particules qu'il mentionne sont fonctionnelles, c'est-à-dire qu'elles ont la structure d'une fonction qui s'applique à un ou plusieurs arguments. L'auteur se fonde essentiellement sur certaines particules de l'allemand, dites modales, comme ja, doch, halt ou wohl. Il oppose au cas des particules celui des interjections (les particules émotionnelles, dans le présent article), qui seraient, selon lui, saturées, autrement dit exprimeraient simplement une émotion, sans avoir une structure fonctionnelle. C'est sur ce point que je me sépare de son analyse, et cela pour une raison relativement triviale : il n'y pas d'émotion " pure » ou «flottante », toute émotion communiquée est émotion à partir de quelque chose. Prenons le cas extrême d'un état intérieur, que rien ne rend manifeste pour un observateur distinct du locuteur. À partir du moment où ce locuteur utilise une interjection, aussi sémantiquement vague qu'elle puisse être, il signale à l'interlocuteur qu'il caractérise «quelque chose » comme justiciable de cette interjection (cf. Kaplan 1999, et, pour une discussion sur la nature indicielle de l'interjection, Kleiber 2006). En l'occurrence il s'agit d'un état intérieur, que personne n'appréhendera peut-être jamais de l'extérieur et qui peut-même être fictif, mais qui demeure en droit distinct de la description (couleur, perspective) sous laquelle le locuteur le présente dans la communication. Ce point m'amène directement à la question de la projection.

\subsection{Projection des particules discursives}

\subsubsection{La composante présuppositionnelle}

Les particules sont donc déictiques et, à ce titre, comme tous les dispositifs indexicaux, elles présupposent l'existence de ce vers quoi elles pointent. Comme je l'ai dit plus haut (§ 2.2.3), il existe une approche anaphorique des présuppositions (Geurts 1999, 46-sq., Van der Sandt 1992). Cette approche assimile les déclencheurs de présupposition à des pointeurs vers le contexte, qu'il soit linguistique (segments de discours antérieurs) ou situationnel (objets, événements) et fait des déclencheurs anaphoriques traditionnels un cas particulier de présupposition. Geurts insiste sur le double fonctionnement de certains déclencheurs de présupposition, qui pointent vers et décrivent d'une certaine manière leur antécédent, c'est-à-dire ce à quoi ils font référence. C'est précisément ce que j'ai mis en évidence à propos des particules dans la section 3.1.2. Il en résulte que l'on peut aussi assimiler les particules aux déclencheurs de présupposition dans le sens de Van der Sandt et Geurts.

Quelles sont les conséquences pour la projection des particules ? Dans la section 2.2.3, j'ai indiqué que l'approche pragmatique en termes de pertinence par rapport à la QUD 
faisait de la projection une propriété dépendante du contexte. Par ailleurs, j'ai avancé que les particules se projetaient obligatoirement, c'est-à-dire qu'elles ne sont jamais affectées par des opérateurs comme la négation, l'interrogation ou les verbaux modaux de possibilité. Dans un exemple comme (15),

(15) A - Paul est venu à la réunion.

B - Tu m'étonnes !

$\mathrm{C}-\mathrm{Tu}$ es sûr? Tu m'étonnes pas.

l'assertion de A introduit ou poursuit une QUD qui peut être la présence de Paul à la réunion, ou, plus largement, un thème qui concerne Paul ou la réunion en question. La réponse de B, qui ne peut subir aucune modification, manifeste que la présence de Paul constitue un événement totalement attendu (contenu sémantique de la particule tu m'étonnes) et pointe vers l'assertion de A. Le contenu sémantique est clairement figé puisqu'il est antiphrastique par rapport à ce qui pourrait constituer le contenu « littéral », tu m'étonnes ! signifiant ici que le locuteur n'est pas étonné par l'assertion de A. La réponse de $\mathrm{C}$, qui pourrait être modifiée (par ex. je ne suis pas étonné), commence par l'expression d'un doute. Dans cette optique, il serait tout à fait pertinent d'indiquer que l'on est surpris par l'assertion et que l'on n'y adhère pas spontanément. Si tu m'étonnes !, comme particule, pouvait être affectée par une négation, on s'attendrait à ce que son contenu sémantique soit inversé, le locuteur C communiquant alors qu'il est étonné par l'assertion. $\mathrm{Si}$ la réponse de $\mathrm{C}$ signifie quelque chose, ce n'est en tout cas pas quelque chose qui se laisse paraphraser par Tu es sûr? Je suis surpris que tu dises ça. Dans la plupart des cas, il n'est même pas possible de construire un exemple où la particule pourrait se combiner avec un opérateur caractéristique pour la projection (négation, interrogation, etc.).

On peut compléter ces remarques en examinant le statut des particules discursives par rapport à la propriété d'effet local (Tonhauser et al. 2013). Un déclencheur de présupposition provoque un effet local lorsque, quand on l'enchâsse dans un opérateur de croyance (penser, estimer, croire que, etc.), la présupposition qu'il déclenche est interprétée comme étant dans la portée de l'opérateur, autrement dit, elle est attribuée au sujet de l'opérateur. Par exemple, dans Paul pense que Marie a cessé de fumer, Paul pense que Marie ne fume pas mais aussi qu'elle a fumé. Paul (le sujet de l'opérateur de croyance penser que) est saisi comme endossant la présupposition du déclencheur cesser de.

Certains déclencheurs, comme cesser de justement, induisent automatiquement cet effet. Dans la terminologie de Tonhauser et al. (2013), ils ont un effet local obligatoire. Pour d'autres déclencheurs, la présence ou non d'un effet local dépend du contexte d'interprétation. Les particules occupent l'autre extrémité du spectre : elles n'ont jamais d'effet local. Par exemple, dans (16), les particules zut ! et quoi ne peuvent être attribuées à Paul.

(16) a. Paul estime que Marie va être élue, zut!

b. Paul estime que Marie va être élue, quoi.

L'effet local semble très proche de ce que Potts (2007) appelle la déplaçabilité, le fait que l'on puisse inscrire le contenu d'un déclencheur dans une perspective éloignée du contexte d'énonciation, par exemple celle d'une croyance, d'un discours indirect, ou d'une description d'événements passés ou futurs. Toutefois, alors que Potts considère que les expressifs en général (les implicatures conventionnelles émotionnelles ou axiologiques) sont non déplaçables par nature, Gutzmann fait valoir que ce comportement n'est pas uniforme et présente la réponse de Potts $^{6}$ à ce problème (Gutzmann 2013, 37-42). D'une manière générale, les expressifs sont centrés sur un énonciateur, qui n'est pas forcément le locuteur, comme en (17a) où l'expressif fichu peut être attribué au locuteur mais aussi à Marie. Lorsque l'on ajoute un opérateur caractéristique de la projection comme en (17b), celle-ci est automatique parce qu'il serait incohérent qu'un énonciateur prenne en charge l'expressif et le mette en doute simultanément. Ainsi, il serait incohérent d'interpréter (17b) 
comme signifiant que Marie prend en charge la dépréciation de son ordinateur (effet de l'expressif) et la met en doute en même temps (effet de la négation). La seule interprétation possible est que Marie ne pense pas que son ordinateur fonctionne mal et que locuteur ou elle-même prend en charge l'expressif dépréciatif.

a. Marie pense que son fichu ordinateur fonctionne mal.

b. Marie ne pense pas que son fichu ordinateur fonctionne mal.

Les expressions centrées sur un énonciateur auront donc une forte tendance à se projeter. Cela vaut notamment pour les particules discursives, qui sont centrées sur un unique énonciateur, source de l'énonciation, c'est-à-dire le locuteur. Les expressions qui ont effet local, ou sont déplaçables, tendront à se projeter si elles sont centrées sur un énonciateur.

Les particules, telles qu'elles sont conçues ici, n'ont pas d'effet local et ne sont pas déplaçables au sens de Potts. Cependant, comme je l'ai expliqué, elles pointent vers des éléments de la situation d'énonciation qui doivent être contemporains de leur énonciation. On ne peut donc les faire se mouvoir à travers le temps ou les perspectives de différents énonciateurs. Elle opèrent $i c i$ et maintenant par rapport à leur apparition physique dans le discours. Le seul énonciateur coïncidant physiquement avec le moment de l'énonciation et qui puisse endosser la valeur sémantique de la particule est le locuteur. C'est donc lui qui est l'énonciateur de référence et sur lequel la particule est centrée, ce qui prédit de fortes propriétés de projection.

\subsubsection{Particules discursives vs connecteurs}

Il est important de distinguer entre la fonction de particule et la fonction de connecteur. Ceci permet d'éclairer la spécificité des particules discursives et de corréler leur capacité de projection et leur fonctionnement énonciatif. Les connecteurs se distinguent des particules par au moins deux propriétés.

(a) Ils relient deux éléments (propositions, segments de discours selon les théories) entre lesquels ils expriment certaines relations (cause, but, conséquence, condition, contraste, etc. $)^{7}$ Ils peuvent à ce titre être qualifiés de présuppositionnels, en ce sens que leur interprétation est dépendante d'un ou plusieurs autre(s) élément(s) dans le discours, élément(s) dont ces connecteurs présupposent l'existence dans le discours (voir la perspective générale de Halliday \& Hasan sur la cohérence, 1976, 4) ${ }^{8}$. Cela explique qu'en général ils ne peuvent pas constituer un énoncé à eux seuls, à la différence de la plupart des particules.

(b) Contrairement aux particules, qui sont toutes ancrées dans le ici et maintenant (ne sont pas déplaçables), certains connecteurs peuvent être déplacés et attribués à un autre énonciateur que le locuteur, comme en (18):

(18) Marie pense que Paul est le président de cette commission et de ce fait ne peut prendre part au vote, mais c'est faux, dans les textes, le président a le droit de vote.

La relation de conséquence marquée par de ce fait est présentée comme prise en charge par Marie. Le déclencheur de ce fait a donc un effet local, ce qui serait impossible avec une particule, comme le montre (19) :

(19) Marie pense que Paul est le président de cette commission et de ce fait, bon, a un devoir de réserve ...

Ici, la particule bon ne peut pas être attribuée à Marie, mais seulement au locuteur qui présente sa formulation a un devoir de réserve comme quelque chose qu'il ne veut ou ne peut pas élaborer.

Les connecteurs ne se projettent pas automatiquement ${ }^{9}$. Dans certains contextes, ils sont sensibles aux opérateurs caractéristiques de la projection. 
A - Paul ne doit pas voter car il est président de la commission.

B - Paul est président mais il n'est pas vrai que du coup il est exclu du

vote, puisque, dans les textes, le président a le statut d'électeur.

En (20), la relation de conséquence invoquée par A est rejetée par B, ce qui montre que le connecteur $d u$ coup ne se projette pas. Dans le détail, la projection ou non des connecteurs est un problème plus complexe, qui ne sera pas développé ici. Le point important est que les connecteurs se projettent de façon moins systématique que les particules. Une explication intuitive de cette différence est que les connecteurs ne sont pas, eux, centrés conventionnellement sur le locuteur, ni sur l'énonciateur.

\section{Conclusion}

Il ressort de cette étude un certain nombre de propriétés relatives aux particules discursives telles qu'elles ont été envisagées. La mise au point sur la question de la projection (section 2) a d'abord montré que la conception classique lexico-grammaticale (opérateur + déclencheur $\rightarrow$ projection) n'était pas en pratique très robuste. Les recherches plus récentes sur la question, dont certaines expérimentales (Tonhauser et al. 2018) et sur corpus (Beaver 2010), ont nuancé le caractère systématique de la projection. De plus, la réflexion de Potts (2005) sur les implicatures conventionnelles a ouvert la liste des déclencheurs à d'autres expressions que les déclencheurs de présupposition ; on y trouve notamment les expressifs, qui partagent certaines propriétés avec les interjections, que j'ai définies ici comme des particules émotionnelles (expressives).

On peut donc résumer l'état actuel des études sur la projection en deux points :

(a) les éléments linguistiques susceptibles de se projeter ne se réduisent pas aux déclencheurs de présupposition (section 2.2.1),

(b) la projection n'est pas une propriété automatique et uniforme, elle dépend de l'opérateur, de l'interprétation et du déclencheur (section 2.2.2).

Cet article contribue à enrichir les deux points précédents.

Concernant (a), je propose d'étendre la classe des déclencheurs de présupposition aux marqueurs discursifs, connecteurs et particules (section 3). En effet, ces marqueurs font référence à un élément du contexte linguistique ou situationnel, qu'il est nécessaire de récupérer pour accéder à une interprétation cohérente. Ceci a pour conséquence que la question de la projection se pose également pour ces marqueurs (section 3.2).

Concernant (b), étant donné le caractère éminemment pragmatique de l'interprétation du discours, on pourrait s'attendre à ce que la projection ou non de ces marqueurs soit relativement variable en fonction du contexte (rôle de la pragmatique). Or, ceci n'est pas vrai pour les particules discursives, qui se caractérisent par une projection systématique. C'est un aspect potentiellement paradoxal et dont il faut rendre compte. J'ai avancé comme explication de cette propriété « en dur » que c'était la subjectivité intrinsèque des particules - elles sont obligatoirement branchées sur le locuteur - qui leur interdisait tout effet local. Cette subjectivité forte vient de leur fonction énonciative particulière : ce à quoi elles réfèrent doit être concomitant de leur propre énonciation. Les connecteurs, qui sont aussi des marqueurs de discours, ne sont, eux, pas soumis à cet arrimage énonciatif et paraissent plus « souples » du point de vue de la projection. Par exemple, en (20) (section 3.2.2), $d u$ coup ne se projette pas dans certains contextes. Une étude détaillée reste à faire pour apprécier les propriétés de projection des connecteurs et leur sensibilité à l'interprétation en contexte.

\section{Remerciements}

Je remercie les deux relecteurs anonymes dont les remarques m'ont permis de clarifier et d'enrichir la première version de cet article. 


\section{Références bibliographiques}

Aijmer, K. (2006). Understanding pragmatic markers. A variational pragmatic approach. Edinburgh : Edinburgh University Press.

Asher, N., Lascarides, A. (2003). Logics of conversation. Cambridge : Cambridge University Press.

Bartkova, K., Dargnat, M., Jouvet, D., Lee, L. (2017). Annotations de particules de discours en français sur une large variété de corpus. In Danlos, L., Fort, K., Guillaume B., Kahane, S. (éd.), Actes de l'atelier "ACor4French - les corpus annotés du français 》,TALN 2017, Orléans, 10-17.

Bastien, A., Bartkova, K., Dargnat, M. (2016). How to be a Discourse Particle ? In Barnes, J., Brugos, A., Shattuck-Hunagel S., Veilleux, N. (eds), Proceedings of Speech and Prosody 2016, Boston : International Speech Communication Association, 858-863.

Beaver, D. (2010). Have you noticed that your belly button lint colour is related to the colour your clothing? In Bäuerle, R., Reyle, U., Zimmermann, E. (eds.), Presuppositions and Discourse: Essays Offered to Hans Kamp. Oxford : Elsevier, 65-99.

Beaver, D., Roberts, C., Simons, M., Tonhauser, J. (2017). Questions under discussion : Where information structure meets projective content. Annual Review of Linguistics, 3, 265-284.

Beaver, D., Clark, B. Z. (2008). Sense and Sensitivity. How Focus Determines Meaning. Chichester : Wiley-Blackwell.

Beeching, K. (2001). Gender, politeness and pragmatic particles in French. Amsterdam: John Benjamins.

Beeching, K. (2016). Pragmatic markers in British English. Meaning in social interaction. Cambridge : Cambridge University Press.

Brémond, C. (2002). Les "petites marques du discours ». Le cas du marqueur méta-discursif bon en français. Thèse de l'Université de Provence.

Chierchia, G., McConnell-Ginet, S. (1990). Meaning and Grammar : An Introduction to Semantics. Cambridge : MIT Press.

Crible, L., Degand, L. (sous presse). Domains and functions: A two-dimensional account of discourse markers. Discours : revue de linguistique, psycholinguistique et informatique.

Dargnat, M. (sous presse). Interjections et particules de discours. In Abeillé, A., Godard, D., Gautier, A. (éd.), Grande Grammaire du Français. Arles : Actes Sud, 17 p.

Dargnat, M., Jayez, J. (sous presse). Presupposition Projection and Main Content. In Abeillé, A., Bonami, O. (eds), Constraint-based Syntax and Semantics. Papers in Honor of Danièle Godard. Stanford : CSLI publications, $26 \mathrm{p}$.

Denturck, E. (2008). Étude des marqueurs discursifs, l'exemple de quoi. Master in de Taal-en Letterkunde. Universiteit Gent.

Dostie, G. (2004). Pragmaticalisation et marqueurs discursifs. Analyse sémantique et traitement lexicographique. Liège : De Boeck/Duculot.

Ducrot, O. (1972). Dire et ne pas dire. Principes de sémantique linguistique. Paris : Hermann.

Ducrot, O. (1984). Le Dire et le dit. Paris : Les Éditions de Minuit.

Fernandez-Vest, J. (1994). Les particules énonciatives dans la construction du discours. Paris : Presses Universitaires de France.

Fischer, K. (ed.) (2006). Approaches to Discourse Particles. Elsevier : Amsterdam.

Fraser, B. (1999). What are discourse markers? Journal of pragmatics, 31(7), 931-952.

Frege, G. (1892). Über Sinn und Bedeutung. Zeitschrift für Philosophie und philosophische Kritik, $100,25-50$.

Geurts, B. (1999). Presuppositions and pronouns. Amsterdam : Elsevier.

Ginzburg, J. (2012). The Interactive Stance. Meaning for Conversation. Oxford : Oxford University Press.

Grieve, J. (1995). Dictionary of contemporary French connectors. Londres : Routledge.

Gutzmann, D. (2013). Expressives and beyond : An introduction to varieties of use-conditional meaning. In Gutzmann, D., Gärtner, H.-M. (eds), Beyond expressives : Explorations in use-conditional meaning. Leiden : Brill, 1-58.

Gutzmann, D. (2015). Use-conditional meaning. Studies in multidimensional semantics. Oxford : Oxford University Press.

Gutzmann, D. (2019). The Grammar of expressivity. Oxford : Oxford University Press.

Halliday, M.A.K., Hasan, R. (1976). Cohesion in English. London : Longman.

Hansen, M.-B. M. (1998). The Function of discourse particles. Amsterdam : John Benjamins. 
Jayez, J. (2010). Projective meaning and attachment. In Logic, Language and Meaning. Revised selected papers of the 17th Amsterdam Colloquium, Amsterdam 2009, $\mathrm{n}^{\circ} 6042$ in Lecture Notes in Artificial Intelligence. Berlin : Springer, 325-334.

Kaplan, R. (1999). The meaning of ouch and oops. Transcription d'une conférence. http://eecoppock.info/PragmaticsSoSe2012/kaplan.pdf

Karttunen, L. (1971). Some observations on factivity. Papers in Linguistics, 4, 55-69.

Karttunen, L. (2016). Presupposition : What went wrong. In Proceedings of Semantics and Linguistic Theory (SALT) 26, 705-731.

Kerbrat-Orecchioni, C. (2009 [1980]). L'énonciation. Paris : Armand Colin.

Kiparsky, P., Kiparsky, C. (1971). Fact. In Steinberg, D. \& Jakobovits, L. (eds.), Semantics. An Interdisciplinary Reader in Philosophy, Linguistics and Psychology. Cambridge: Cambridge University Press, 345-69.

Kleiber, G. (2006). Sémiotique de l'interjection. Langages, 161, 10-23.

Langendoen, D. T., Savin, H. B. (1971). The projection problem for presupposition. In Fillmore, J., Langendoen, D. T. (eds), Studies in Linguictic Semantics. New York/Chicago/San Francisco: Holt, Rinehart and Winston, 54-60.

Mann, W. \& Thompson, S. (1988). Rhetorical Structure Theory : Toward a functional theory of text organization. Text, 8(31), 243-281.

Métrich, R., Faucher, E., Courdier, G. (2002). Invariables difficiles, Dictionnaire allemand-français des particules, connecteurs, interjections et autres mots de la communication. Nancy: Groupe de lexicographie franco-allemande de l'Université de Nancy 2 et ATILF.

Morgan, J. (1969). On the treatment of presupposition in transformational grammar. Proceedings of the Chicago Linguistics Society, 5, 167-177.

Paillard, D. (1998). Les mots du discours comme mots de langue. Le Gré des langues, 14, 10-41.

Peters, S. (2016). Speakers commitments : presupposition. In $n$ Proceedings of Semantics and Linguistic Theory (SALT) 26, 1083-1098.

Potts, C. (2005). The Logic of conventional implicatures. Oxford : Oxford University Press.

Potts, C. (2007). The expressive dimension. Theoretical Linguistics, 33, 255-268.

Renkema, J. (2009). The Texture of Discourse. Amsterdam : John Benjamins.

Roberts, C. (2012 [1998]). Information structure in discourse : Towards an integrated formal theory of pragmatics. Semantics and Pragmatics, 5(6), 1-69.

Roze, C. (2009). Base lexicale des connecteurs discursifs du français. Mémoire de M2 recherche en linguistique informatique de l'Université Paris 7.

Simons, M. (2007). Observations on embedding verbs, evidentiality, and presuppositions. Lingua, $117,1034-1056$.

Simons, M., Tonhauser, J., Beaver ,D., Roberts, C. (2011). What projects and why. In Li, N., Lutz, D. (eds), Semantics and Linguistic Theory (SALT) 20, eLanguage, 309-327.

Simons, M., Beaver, D., Roberts, C., Tonhauser, J. (2017). The best question : Explaining the projection behavior for factives. Discourse Processes, 54 (3), 187-206.

Stalnaker, R. C. (1974). Pragmatic presuppositions. In Munitz, M., Unger, P. (eds), Semantics and Philosophy. New York : New York University Press, 197-214.

Tonhauser J., Beaver D., Roberts C., Simons M. (2013). Toward a taxonomy of projective content. Language, 89, 66-109.

Tonhauser, J., Beaver, D.I. \& Degen, J. (2018). How projective is projective content? Gradience in projectivity and at-issueness. Journal of Semantics, 35, 495-542.

Traugott, E.-C. (1982). From propositionnal to textual and expressive meanings : somme semanticpragmatic aspects of grammaticalization. In Lehmann, W. P., Malkiel, Y. (eds), Perspectives on historical linguistics. Amsterdam : John Benjamins, 245-272.

Van der Sandt, R.A. (1992). Presupposition projection as anaphora resolution. Journal of Semantics, 9, 333-377.

Vincent, D., Sankoff, D. (1992). Punctors : a pragmatic variable. Language Variation and Change, 4, 205-216.

Vincent D. (1993). Les ponctuants de la langue et autres mots du discours. Québec: Nuit Blanche Éditeur.

Wharton, T. (2003). Interjections, language and the showing-saying continuum. Pragmatics and Cognition, 11.1, 39-91. 
${ }^{1}$ Par souci de cohérence, je conserve ici le sigle anglais QUD en référence à Question Under Discussion.

${ }^{2}$ A ma connaissance, le terme de projection a été utilisé pour la première fois par Morgan (1969) dans le cadre de la grammaire transformationnelle. Il renvoie à l'idée que la présupposition remonte (se projette) au-dessus de l'arbre syntaxique de la phrase.

${ }^{3}$ Potts introduit les expressifs dans la section 2.3.2 (2005, 16-22). Il fait notamment référence à des éléments lexicaux émotionnellement et/ou axiologiquement marqués, qui relèvent de la subjectivité au sens large. Il cible les items marquant un haut degré émotionnel, l'orientation de cette émotion étant variable. Son apport principal est classificatoire : il fait de ces items des déclencheurs d'implicature conventionnelle.

La composante expressive de certains lexèmes est aussi abordée dans les études sur l'énonciation et la subjectivité. Voir par exemple, pour le français, les travaux de Kerbrat-Orecchioni (2009 [1980], chapitre 2 « De la subjectivité dans le langage : quelques-uns de ses lieux d'inscription »).

${ }^{4}$ Sont désignés ainsi les verbes qui présupposent la vérité de la proposition complément (qui la présentent comme un fait acquis). Par exemple, Marie sait que Paul fume présente comme vraie la proposition Paul fume.

${ }^{5}$ Un relecteur anonyme m'a fait remarquer à juste titre qu'il y avait aussi une valeur épistémique possible, point barre soulignant le statut indiscutable de l'information pour le locuteur.

${ }^{6}$ Pour l'essentiel, cette réponse consiste à inclure dans le contexte d'énonciation un juge (dans une perspective à la Ducrot, un énonciateur contextuellement saillant), qui peut être distinct du locuteur, et qui prend en charge le contenu de l'implicature. Par exemple, des expressions comme le salaud, le traître, le génie, etc., pourraient dénoter ce qu'un juge présent dans le contexte d'énonciation pense de tel ou tel personnage. Le but de la réponse de Potts est de permettre une certaine variabilité (effet local, déplaçabilité) tout en ancrant sa source dans le contexte d'énonciation, ce qui préserve le caractère d'implicature, distincte des autres éléments de contenu qui, eux, contribuent à répondre à la QUD.

${ }^{7}$ Pour une liste des connecteurs en français, voir le dictionnaire de Grieve (1995) et le relevé de Roze (2009). Pour une typologie des relations de discours, voir la description de Asher \& Lascarides (2003), la synthèse de Crible \& Degand (sous presse), le système de Renkema (2009) ainsi que les recherches autour de la Rhetorical Structure Theory (https://www.sfu.ca/rst, Mann \& Thompson 1988).

${ }^{8}$ Je fais un emploi étendu de la notion de présupposition, habituellement appliquée à des contenus présentés comme vrais. Ici, dans le cas des connecteurs, et des particules, ce qui est présupposé est l'existence d'un segment de discours ou d'un événement, sans que que soit forcément évaluée la vérité de son contenu.

${ }^{9}$ Je ne considère pas ici les connecteurs qui contribuent aux conditions de vérité de la phrase, par exemple les temporels (avant que, après que, lorsque, au moment où, etc.), le causal (parce que) et les conditionnels (si, au cas où, etc.). Ces connecteurs et la proposition qu'ils introduisent sont d'ailleurs insérables dans une clivée, et ils tombent sous la portée des opérateurs comme la négation, l'interrogation ou les modaux de possibilité. 